\title{
Novos compostos dietéticos com propriedades anticarcinogênicas
}

\author{
New dietetic compounds with anticarcinogenic properties
}

Carlos Kusano Bucalen Ferrari' e Elizabeth Aparecida Ferraz da Silva Torres ${ }^{2}$

\begin{abstract}
Resumo
Esta revisão teve como objetivo descrever e analisar a importância de compostos alimentares com propriedades anticarcinogênicas. M inerais, como o Selênio e o Cálcio, e compostos presentes em chás (catequinas), cítricos (terpenos), no caqui, no leite, em peixes, em uvas e vinhos (fenólicos), na soja e derivados (isoflavonóides) apresentam comprovados efeitos anticarcinogênicos in vitro e in vivo, constituindo promissores alimentos funcionais para a prevenção do câncer.
\end{abstract}

Palavras-chave: carotenóides; fenólicos; terpenos; isoflavonóides; anticarcinogênicos.

\section{Abstract}

To describe and analyse the importance of food compounds with anticarcinogenic properties was the objective of this review. Minerals, as Selenium and Calcium, and compounds present in teas (catechins), citrics (terpenes), persimmon, milk, fish, grapes and wine (phenolics), soy beans and its products (isoflavonoids) have confirmed anticarcinogenic effects in vitro and in vivo, constituting promising functional foods to cancer prevention.

Key words carotenoids, phenolics, terpenes, isoflavonoids, anticarcinogenic agents

${ }^{1}$ M estre e D outorando em Saúde Pública - D epartamento de N utrição da Faculdade de Saúde Pública da Universidade de São Paulo.

2Profa. D outora e Livre-D ocente - D epartamento de N utrição da Faculdade de Saúde Pública da U niversidade de São Paulo (USP). Enviar correspondência para E.A.F.S.T. Av. Dr. Arnaldo 715, 20 andar; 01246-904 Cerqueira César, SP - Brasil. e-mail: eatorres@usp.br

Pesquisas financiadas pelo CN Pq e FAPESP

Recebido em janeiro de 2002. 


\section{INTRODUÇÃO}

Embora de origens e causas ainda não muito esclarecidas, as neoplasias surgem devido a mutações genéticas, espontâneas ou induzidas por agentes patogênicos [metais, radiações, radicais livres do oxigênio, inflamações crônicas e xenobióticos (cigarro, álcool, pesticidas, etc), dentre outros], que promovem desordens no ciclo celular, ocorrendo excesso nas taxas de proliferação e deficiência nas taxas de morte celular, culminando com a formação de agrupamentos de clones de células neoplásicas, os tumores.

0 principal grupo de agentes indutores da carcinogênese é representado pelas espécies reativas do oxigênio e nitrogênio, outros radicais livres e produtos da peroxidação lipídica (PL) que induzem diversas injúrias celulares e nucleares. ${ }^{1-6}$

D iversos estudos têm comprovado, sem dúvida alguma, que existe uma forte associação inversa (ou negativa) entre 0 consumo de frutas e verduras e 0 risco de diversos tipos de cânceres e outras causas de morbi-mortalidade. ${ }^{6-8}$

Assim, o homem há várias décadas vem isolando compostos presentes em alimentos de origem vegetal para testá-los como possíveis agentes anticarcinogênicos.

Entretanto, dois importantes estudos clínicos controlados e randomizados revelaram que a suplementação de fumantes com $\beta$-caroteno aumentou o risco de câncer de pulmão e o uso do tocoferol também não trouxe benefícios aos fumantes. ${ }^{9}$

Porém, já existem sólidas evidências, ${ }^{10}$ para sustentar a tese que os carotenóides provenientes da dieta (e não de suplementos) podem diminuir o risco de câncer pulmonar e de outras patologias, tanto que os derivados da vitamina $A$, os retinóides, são promissores fármacos antineoplásicos. ${ }^{11}$

Além disso, outros estudos clínicos controlados e randomizados também não evidenciaram qualquer efeito da suplementação com vitaminas antioxidantes sobre a oxidação do DNA de células orais e mononucleares oriundas de fumantes inveterados. ${ }^{12}$

Percebe-se, então, que na verdade não são os compostos isolados e ingeridos sob a forma de suplementos que podem diminuir o risco de câncer, mas sim uma dieta rica em substâncias anticarcinogênicas, incluindo novos compostos recentemente isolados, que ainda se encontram em estudo pelo homem.

$O \mathrm{~s}$ alimentos contendo agentes para a quimioprevenção do câncer constituem um dos principais grupos de alimentos com propriedades funcionais, conhecidos também como nutracêuticos ou fármaco-alimentos. ${ }^{13}$

0 objetivo deste estudo foi realizar uma breve introdução sobre as funções destes "novos" compostos anticarcinogênicos presentes em alimentos.

\section{EFEITOS ANTITUMORAIS DOS ALIMENTOS}

D iversas investigações epidemiológicas (populacionais) e experimentais (in vivo ou em animais e in vitro ou em culturas de células), têm demonstrado que os antioxidantes podem diminuir o risco de diversos tipos de cânceres [ácido ascórbico, fitoestrógenos da soja, carotenóides ( $\alpha$-caroteno, $\beta$-caroteno, luteína, zeaxantina)].6-8,10,14,15

0 principal grupo de agentes inibidores da carcinogênese é representado por antioxidantes, bloqueadores de radicais livres. Além destes, também temos os indutores da morte celular programada (apoptose), os inibidores das enzimas do citocromo P450 (responsável pelo metabolismo de drogas, cuja ativação leva à formação de radicais livres carcinogênicos), outros inibidores enzimáticos, inibidores da angiogênese (neoformação de vasos sanguíneos, necessária para a disseminação dos tumores através das metástases), antagonistas de fatores de crescimento, hormônios e agentes reparadores de lesões ao D N A. ${ }^{11,16}$

$\mathrm{Na}$ Tabela 1, estão agrupadas substâncias com efeitos anticarcinogênicos já comprovados e suas fontes dietéticas. ${ }^{11}$

\section{OS ALIMENTOS E SEUS COMPOSTOS ANTICARCINOGÊNICOS}

\section{MINERAIS}

Elevados teores de Selênio em brócolis foram responsáveis pela diminuição das taxas de câncer de cólon em ratos, ${ }_{17}^{17}$ corroborando com estudos anteriores quevêm demonstrando 
o papel deste elemento na prevenção do câncer de próstata. ${ }^{11} \mathrm{O} \mathrm{s}$ mecanismos anticarcinogênicos desempenhados pelo Selênio ${ }^{18}$ encontram-se na Tabela 1.

0 cálcio também desempenha um importante papel antineoplásico. No tubo digestivo, este mineral inibe a proliferação e aumenta a diferenciação de células intestinais, inibe a ativação da enzima ornitinadescarboxilase $(O D C)^{19}$ e promove a ativação da apoptose de células tumorais, através tanto da ativação das enzimas caspases, que promovem a desestruturação nuclear e das organelas, ${ }^{20}$ quanto da ativação das transglutaminases. ${ }^{21}$

O utros minerais, como $M$ anganês, $M$ agnésio, $Z$ inco, Cobre e $M$ olibdênio podem proteger o DNA, bem como apresentar funções imuno-moduladoras, mas pouco se sabe a respeito de propriedades antineoplásicas específicas.

Tabela 1. Mecanismos e fontes de agentes anticarcinogênicos naturais.

\begin{tabular}{|c|c|c|}
\hline $\operatorname{nat} \mathbf{k}$ & Hecariemas & Fan les dimen bres: \\
\hline Nido Bím & Comrç̧ de repax na DWh & 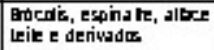 \\
\hline فالمة & $\begin{array}{l}\text { hdue a apop bes; liveres xs } \\
\text { sides bilixes }\end{array}$ & \\
\hline ca kquinz & 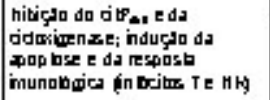 & ahy (Nerde e preb) \\
\hline $\begin{array}{l}\text { Fanif- } \\
\text { iso ofocisnabs }\end{array}$ & 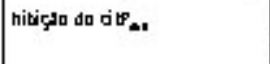 & $\begin{array}{l}\text { Orudtras procolia } \\
\text { muve- brj) }\end{array}$ \\
\hline hdale-3restind & 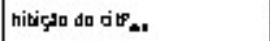 & Stattis cud term \\
\hline 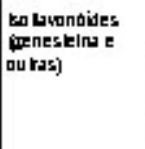 & 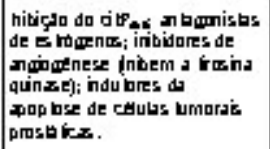 & Sqi e drikstas \\
\hline Limaneng & 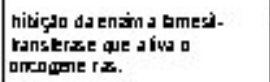 & timse hubstrics \\
\hline Sumina $D$ & 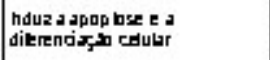 & 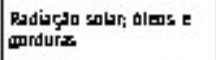 \\
\hline $\begin{array}{l}\text { Uibmina Ee } \\
\text { Selenio }\end{array}$ & 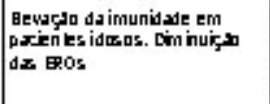 & 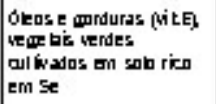 \\
\hline me tintides & 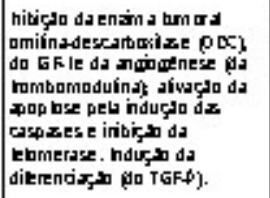 & 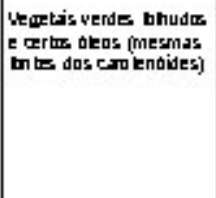 \\
\hline Restera mite & 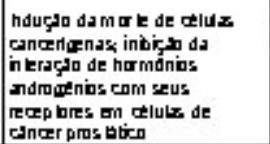 & 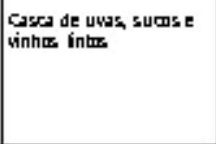 \\
\hline
\end{tabular}

Fontes: referências' ${ }^{11,18,31,32,40}$

\section{FRUTAS CÍTRICAS}

Ricas em vitaminas, fibras e potássio, os cítricos também apresentam carotenóides, principalmente a $\beta$-criptoxantina, ${ }^{22}$ e diversos terpenos, como monoterpenos, limonenos (D limoneno, limonina, 17- $\beta$-D-glicopiranosídeo, nomilina), flavonóides, carotenóides eácido hidroxicinâmico, qualificando-os para a prevenção de várias doenças, incluindo 0 câncer. ${ }^{23}$ Tais limonóides presentes nas frutas cítricas (cidra, laranja elimão) têm como principal mecanismo de ação a indução da G lutationa-S-Transferase (GST), enzima que promove a conjugação de carcinógenos (substâncias eletrofílicas) que sofreram prévia bioativação pelas enzimas de fase I do metabolismo de xenobióticos no interior do citocromo P450, tendo como conseqüência a diminuição da toxicidade das substâncias mutagênicas. ${ }^{24,25}$

0 extrato de abacaxi (Ananas comosus M err.) contém bromelina, enzima que apresentou atividade antineoplásica em três doses testadas $(0,4 \mathrm{mg} / \mathrm{ml}, 0,6 \mathrm{mg} / \mathrm{ml} \mathrm{e} 0,8 \mathrm{mg} /$ $\mathrm{ml}$ ). A maior dose inibiu em $70 \%$ crescimento da linhagem de células tumorais ascíticas (MCA-1) e em 90\% o desenvolvimento das linhagens carcinoma pulmonar de Lewis (LLC) e linfoma (YC-8). ${ }^{26}$

\section{FRUTAS E VEGETAIS VERMELHOS OU ROXOS (E SUCOS E VINHOS)}

Os principais pigmentos que dão a cor vermelho-arroxeada às uvas e jabuticaba são os flavonóides antocianina e quercetina, 0 carotenóide licopeno e certos ácidos orgânicos. Tratam-se de compostos fenólicos com elevada atividade antioxidante encontrados em diversos alimentos ${ }^{22,27,28}$ (Tabela 2).

Q uercitina, rutina, luteolina, mirecetina, ácido tânico e catequina protegem o D N A de lesões induzidas por espécies reativas do oxigênio. ${ }^{29} 0$ utros polifenólicos importantes são representados pelo grupo das catequinas.

0 caqui (D iospyros kaki L.) contém alto teor de polifenólicos que podem diminuir a sobrevivência de células neoplásicas. N esta perspectiva, Achiwa et al ${ }^{30}$ observaram que os polifenólicos do caqui inibiram em $10 \%$ a $20 \%$ a atividade da ornitina-descarboxilase, responsável pela síntese de poliaminas que 
favorecem a proliferação celular. Além dissso, embora a catequina e a epicatequina tenham sido pouco efetivas, os demais polifenólicos (do extrato puro de caqui, o galato de epicatequina, a epigalocatequina e 0 epigalocatequinagalato) induziram efetivamente a apoptose de células de leucemia linfocítica humana.

Os fenólicos, incluindo o resveratrole, apresentam também potentes efeitos anticarcinogênicos. ${ }^{15,25}$ Aliás, o resveratrole (trihidroxi-estilbeno), presente nas cascas de uvas, tornando-se, pois, concentrado nos vinhos tintos, apresenta capacidade antitumoral através da indução da morte de células neoplásicas. ${ }^{31} 0$ resveratrole também foi capaz de inibir a atividade de receptores para hormônios andrógenos em células tumorais prostáticas. ${ }^{32}$

Produtos derivados de tomate (pasta, suco, purê e ketchup) apresentam elevados teores de carotenóides, especialmente de licopeno, ${ }^{22}$ 0 que Ihes confere 0 status de potentes alimentos funcionais anticancerígenos, capazes de prevenir o câncer de próstata no homem, ${ }^{15}$ bem como outros tumores. N este sentido, um estudo prospectivo de coorte, nos EUA, observou uma relação inversa entre os níveis plasmáticos de licopeno e o risco de câncer de próstata. ${ }^{33}$

Em um estudo de caso-controle realizado em São Paulo, cujo objetivo era verificar a associação entre o consumo de alimentos fontes de $\beta$-caroteno e vitamina $C$ e câncer de mama, observou-se uma correlação inversa apenas para o consumo de goiaba, cujo resultado foi uma $R$ azão dos Produtos Cruzados (RPC) de 0,32 (IC: 95\%, 0,12-0,88 e $p=0,033$ ), significando uma diminuição de cerca de $68 \%$ no risco daquele câncer. ${ }^{34}$ É provável que a presença de outros anticarcinogênicos (flavonóides) também tenha sido responsável por tal efeito.

0 alho, que também pode apresentar coloração arroxeada, promove a elevação das enzimas GST e GSH, diminuindo os níveis biológicos de radicais livres. ${ }^{25}$
Tabela 2. Principais carotenóides e flavonóides e suas fontes alimentares.

\begin{tabular}{|c|c|}
\hline Composto & Fonte \\
\hline 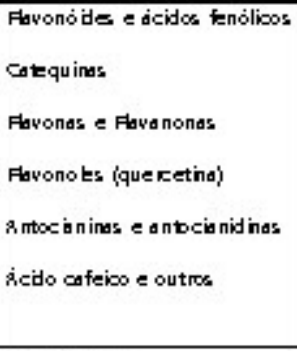 & 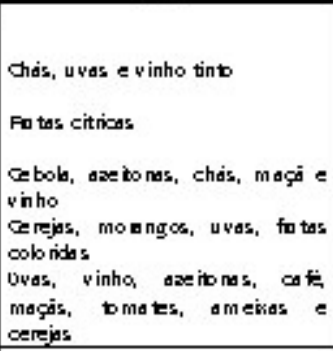 \\
\hline $\begin{array}{l}\text { Gotenöide } \\
\text { arcoroteno e } \beta \text {-coroteno } \\
\text { bopeno } \\
\text { seaxantina } \\
\text { criptontina }\end{array}$ & 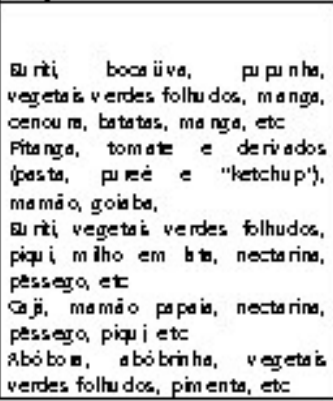 \\
\hline
\end{tabular}

Fontes: referências $22,27,28$

\section{FRUTAS ALARANJADAS OU AMARELADAS}

O s carotenóides são responsáveis por este tipo de coloração e por diversas atividades biológicas, tais como: ${ }^{27}$

- remoção do oxigênio singlete;

- remoção de radicais peroxila;

- modulação do metabolismo de carcinógenos;

- inibição da proliferação celular;

- aumento da diferenciação celular (retinóides);

- estimulação da comunicação intercelular;

- aumento da resposta imunológica; e

- capacidade de filtrar a luz azul.

Sabe-se que o $\beta$-caroteno é capaz de proteger o DNA contra a oxidação. ${ }^{35}$

\section{CAFÉ}

$H$ á indícios que o café também poderia prevenir a oxidação do D N A. Vieira et al ${ }^{36}$ demonstraram que a xantina foi eficaz na inibição da oxidação da adenina e guanosina, efeito mediado através da redução dos radicais Ade. e Gua., respectivamente. Ao contrário, a teobromina e a cafeína não apresentaram efeito; embora a teofilina e a paraxantina tenham sido capazes tão somente de proteger as bases de Adenina.

Revisando os fatores de risco para câncer de boca e faringe, Leite e Koifman ${ }^{37}$ descreveram que certos estudos observaram 
efeitos protetores do café, chá e leite, embora outros não tenham evidenciado tais efeitos.

\section{CRUCífERAS}

Este grupo, em que se encontram 0 repolho, a couve de Bruxelas, a couve-flor e os brócolis, é rico em isotiocianatos, substâncias reconhecidamente anticancerígenas, tanto pela morte tumoral, quanto pela ativação de enzimas detoxificantes do metabolismo de xenobióticos. ${ }^{24,25}$

\section{SOJA E DERIVADOS}

Um grama de soja contém cerca de 1mg de isoflavonas, substâncias antioxidantes com efeitos antitumorais e hipolipemiantes. Estima-se que no Japão o consumo diário de isoflavonas varie de 25 a $50 \mathrm{mg}$. Concentrados de soja em pó podem conter até $160 \mathrm{mg}$ de isoflavonas por porção, valor muito superior ao assumido como seguro (50mg de isoflavonas/dia). ${ }^{3,8}$

Um estudo de caso-controle em Xangai, $C$ hina, observou uma relação inversa entre 0 consumo de alimentos ricos em soja na adolescência e o posterior risco de câncer. Com o ajuste para outros fatores de risco conhecidos, as razões dos produtos cruzados para o consumo total de produtos de soja (tofu, leite e outros) foram de 0,75, 0,69, 0,69 e 0,51 , para os quintis 2 a 5 , respectivamente. $^{39}$

A genesteína (5,7,4'-trihidroxi-isoflavona), foi capaz de promover a apoptose de células de carcinoma da próstata; porém, tal efeito se deve a doses muito superiores àquelas encontradas numa dieta à base de soja. ${ }^{40}$ O utros mecanismos anticarcinogênicos da soja encontram-se na Tabela 1.

\section{CHÁS ORIENTAIS (VERDE E PRETO)}

Os polifenólicos de chás, também encontrados no caqui, desempenham importante papel na prevenção do câncer em países do extremo 0 riente. ${ }^{15} \mathrm{H}$ á fortes evidências demonstrando que as catequinas dos chás (epigalocatequina, galato de catequina, catequina, epicatequina, thearubigina, flavinas, etc), especialmente do verde, apresentam efeitos anticancerígenos, ${ }^{41}$ apresentando sinergismo entre os diversos compostos, inclusive com 0 tamoxifeno. ${ }^{42}$
Um estudo em Pelotas, Rio Grande do Sul, demonstrou que a prevalência de lesões pré-cancerígenas foi 2,2 vezes maior em bebedores de mate quente, 0 chimarrão, em relação aos não bebedores. ${ }^{43}$ O utros estudos epidemiológicos sobre a ingestão de chás em populações brasileiras necessitam ser realizados.

\section{Peixes, laticínIOS e OUtRos}

0 consumo de óleo de peixe, que contém ácidos graxos $\beta$-3, diminui a proliferação de células retais. ${ }^{44}$ Ademais, ratos que ingeriram óleo de peixe tiveram aumento nas taxas de diferenciação e apoptose, mas sem diminuição da proliferação, de células de carcinoma de cólon de ratos induzido pelo carcinógeno azoximetano (AOM). ${ }^{45}$

$\mathrm{N}$ um estudo de caso-controle no $\mathrm{H}$ avaí (EUA), não foi observada associação entre consumo de peixe e risco de câncer de colón e reto. ${ }^{46}$ Entretanto, Estève et al ${ }^{47}$ num estudo multicêntrico de caso-controle, já haviam sugerido que o elevado consumo de peixe diminuía o risco de câncer de faringe (superior e inferior), mas não de endofaringe.

Em outro estudo de caso-controle, realizado em Milão, Fernandez et $a^{48}$ compararam o maior quintil de consumo de peixe com o menor e observaram diminuição do risco de câncer orofaringeal $(R P C=0,5)$. A tendência se repetiu em relação à ingestão de peixe e 0 risco dos cânceres de laringe ( $R P C=0,7)$, esôfago ( $R P C=0,6)$, estômago $(R P C=0,7)$, cólon $(R P C=0,6)$, reto $(R P C=$ $0,5)$, pâncreas ( $R P C=0,7)$, endométrio $(R P C=0,8)$, ovário $(R P C=0,7)$ e mieloma múltiplo (RPC $=0,5)$.

0 ácido linoleico conjugado, ALC (18:2), presente no leite, laticínios, na carne de ruminantes eno pescado (em menores teores), apresenta elevado efeito anticarcinogênico. Ratos alimentados com ALC a 1,5\% tiveram diminuição de $50 \%$ na incidência de tumores mamários induzidos pelo 7,12dimetilbenz[a]antraceno (D M BA). Além disso, - ALC também diminui o risco de neoplasias induzidas pela metilnitrosouréia (M N U) epor quinolinas. ${ }^{49}$

Todavia, apesar da sugestão da existência de fatores protetores no leite e derivados, observou-se num estudo que havia uma 
associação positiva entre consumo de manteiga e risco de cânceres da laringe e hipofaringe, ${ }^{47}$ certamente influenciada pela presença de elevado teor de gorduras suscetíveis à formação de produtos tóxicos originários da peroxidação dos lipídios. ${ }^{6}$

A curcumina do curry indiano foi capaz de inibir a angiogênese induzida pela fator de crescimento de fibroblastos-2 (FGF-2), propriedade importante para a diminuição da capacidade de formação de metástases neoplásicas. ${ }^{50}$

\section{DISCUSSÃO E CONCLUSÃO}

Apesar das evidências epidemiológicas e laboratoriais, as propriedades anticarcinogênicas de minerais, do abacaxi, do café, do caqui, dos chás, de cítricos, do leite, de peixes, de uvas e vinhos e da soja e derivados (dentre outros) devem ser estudadas no país e na América Latina, uma vez que há diferenças entre as populações, bem como entre as variedades de alimentos ao redor do globo terrestre. Estudos de Epidemiologia $\mathrm{N}$ utricional poderão nos dar pistas sobre possíveis associações entre alimentos (e seus compostos) e proteção frente aos cânceres.

\section{REFERÊNCIAS BIBLIOGRÁFICAS}

1. Esterbauer H. Cytotoxicity and genotoxicity of lipid-oxidation products. Am J Clin N utr 1993;57(Suppl):779-86.

2. H alliwell B. Radicales libres, antioxidantes y enfermedad humana: curiosidad, causa 0 consecuencia? Lancet [edición española] 1994;26:109-13.

3. Gutteridge JM . Lipid peroxidation and antioxidants as biomarkers of tissuedamage. Clin Chem 1995;41:1819-28.

4. M edeiros M HG, Loureiro APM, Carvalho VM . Lesões em D N A produzidaspor produtos secundários da peroxidação lipídica. Rev M ed 1996;75:16-25.

5. Ferrari CKB. O xidação lipídica em alimentose sistemas biológicos: mecanismos gerais e conseqüências nutricionaise patológicas. Rev N utr 1998;11:3-14.

6. Ferrari CKB. O xidativestress pathophysiology: searching for an effective antioxidant protection. Int M ed J 2001;8:175-84.
7. Flagg EW, C oates RJ, G reenberg RS. Epidemiologic studies of antioxidants and cancer in humans. J Am C oll N utr 1995;14: 419-27.

8. Weisburger JH. Eat to live, not liveto eat. $\mathrm{Nu}$ utrition 2000;16: 767-73.

9. Antioxidants: nutritional panacea or Pandora's box? Food Technol 1996;50(11):79.

10. Zhang S, H unter DJ, Forman M R, Rosner BA, Speizer FE, Colditz GA, M anson JE, $H$ ankison SE, Willett W C. D ietary carotenoids and vitamins $A, C$, and $E$ and risk of breast cancer. J $N$ atl $C$ ancer Inst 1999;91:547-56.

11. Kell off GJ, Crowell JA, Steele VE, Lubet RA, Boone CW, M aloneWA, H awk ET, Lieberman R, Lawrence J A, Kopelovich L, Ali I, Viner JL, Sigman CC. Progress in cancer chemoprevention. Ann N Y Acad Sci 1999;889:1-13.

12. Jacobson JS, Begg M D , Wang LW, Wang $Q$, Agarwal M, N orkusE, Singh VN , Young T-L, Yang D, Santella R M . Effects of a 6-month vitamin intervention on D N A damagein heavy smokers. Cancer Epidemiol Biomark Prev 2000;9:1303-11.

13. Bloch A, Thomson CA. Position of the American D ietetic Association: phytochemical sand functional foods. J Am Dietet Assoc 1995;95:493-6.

14. Kleiner SM . D efense plants: foods that fight disease. Physic sportsmed [serial online] 1997 [cited 1998 Sept 28]; 25(12). Availablefrom: URL: http://www.physsportsmed.com/issues/ 1997/12dec/defense.htm

15. Weisburger JH. M echanisms of action of antioxidants as exemplified in vegetables, tomatoes and tea. Food Chem Toxicol 1999;37:943-8.

16. H alliwell B. Establishing the significance and optimal intake of dietary antioxidants: the biomarker concept. N utr Rev 1999;57:104-13.

17. Finley $J W, D$ avis $C D$, Feng Y. Selenium from high Selenium broccoli protects rats from coIon cancer. J N utr 2000;130:2384-9.

18. Schrauzer GN . Anticarcinogenic effects of Selenium. Cel M ol LifeSci 2000;57:1864-73.

19. Lipkin M . Preclinical and early human studies of calcium and colon cancer prevention. Ann N Y Acad Sci 1999;889:120-7.

20. Ferrari CKB. F ree radicals, lipid peroxidation and antioxidants in apoptosis: implications in cancer, cardiovascular and neurological diseases. Biologia 2000;55(6):579-88.

21. Fesus L, Madi A, Balajthy $Z$, Szondy $Z$. Transglutaminase induction by various cell 
death and apoptosis pathways. Experientia 1996;52:942-8.

22. Rodriguez-Amaya D B. Latin American food sources of carotenoids. Arch Latinoamer N utr 1999;49(Suppl 1):74-84.

23. EconomosC, Clay W D. N utritional and health benefits of citrus fruits. Food N utr Agricult 1999;24:11-8.

24. Lampe JW. H ealth effects of vegetables and fruit: assessing mechanisms of action in human experimental studies. Am J Clin Nutr 1999;70(Suppl):475-90.

25. Sgarbieri VC, Pacheco M T B. Alimentos funcionais fisiológicos. Braz J Food Technol 1999;2:7-19.

26. Taussig SJ, Szekerczes], Batkin S. Inhibition of tumour growth in vitro by bromelain, an extract of the pineappleplant (Ananas comosus). Planta M ed 1985;51:538-9.

27. O Ison JA. C arotenoidsand human health. Arch Latinoamer N utr 1999;49(Suppl 1):7-11.

28. Croft KD. Thechemistry and biological effects of flavonoidsand phenolic acids. Ann N Y Acad Sci 1998;854:435-42.

29. Ángel M H, González EAP. Plantas que contienen polifenoles. Antioxidantes dentro del estilo de vida. Rev Cubana Invest Biomed 1999;18:12-4.

30. AchiwaY, H ibasami H , Katsuzaki H , Imai K, Komiya T. Inhibitory effects of persimmon (D iospyroskaki) extract and related polyphenol compounds on growth of human lymphoid leukemia cells. Biosci Biotech Biochem 1997;61:1099-101.

31. Soleas G , Grass L, Josephy D , D iamandisEP. Resveratrol, a red wine constituent, has anticarcinogenic properties. Clin Biochem 2000;33:240.

32. M itchell SH, Zhu W, Young CYF. Resveratrol inhibits the expression and function of theandrogen receptor in $\mathrm{LN} C$ aP prostatecancer cells. Cancer Res 1999;59:5892-5.

33. Gann PH, M a J, Giovannucchi E, W illet W, Sacks FM, $\mathrm{H}$ ennekens $\mathrm{CH}$, Stampfer MJ. Lower prostatecancer risk in men with elevated plasma lycopenelevels: results of a prospective study. Cancer Res 1999;59:1225-30.

34. Lima FEL de. Avaliação do consumo de alimentosfontedeBeta-caroteno evitaminaC em relação ao câncer de mama: estudo preliminar [dissertação]. São Paulo: Faculdade deSaúdePúblicadaUniversidadedeSão Paulo; 2000.
35. U megaki K, Ikegami S, InoueK, IchikawaT, Kobayashi S, Soeno N, Tomabechi K. Beta-carotene prevents $\mathrm{x}$-ray induction of micronuclei in human lymphocytes. Am J Clin N utr 1994;59:409-12.

36. VieiraAJSC, Telo JP, PereiraH F, Patrocínio PF, Dias RM B. Antioxidant effect of naturally occuring xanthines on theoxidativedamage of D N A bases. J Chim Phys 1999;96:116-23.

37. LeiteICG, K oifman S. Revisão dos fatores de risco para o câncer de boca efaringe. Rev Bras Cancerol 1998:44:317-25.

38. Gibaldi M . Are phytoestrogens a "natural alternative" to estrogen replacement therapy? West J M ed 2001;173:273.

39. Shu XO , Jin F, D ai Q, Wen W, Potter JD , Kushi L, Ruan Z, G ao Y-T, Zheng W. Soyfood intake during adolescence and subsequent risk of breast cancer among chinese women. $C$ ancer Epidemiol Biomark Prev 2001;10:483-8.

40. KyleE, N eckensL, Takimoto C, Curt G, Bergan R. G enistein-induced apoptosis of prostatecancer cells is preceded by a specific decrease in focal adhesion kinase. Mol Pharmacol 1997;51:193-200.

41. M ukhtar H , Ahmad N . Tea polyphenols: prevention of cancer and optimizing health. Am J Clin N utr 2000;71:1698S-702S.

42. SuganumaM , O kabeS, Kai Y, SueokaN, Sueoka E, Fujiki H . Epigallocatechin G allate with (-) Epicatechin, Sulindac, or Tamoxifen on cancer-preventiveactivity in the human lung cancer cell line PC -9. C ancer Res 1999;59:44-7.

43. M uñozN , VictoraCG , Crespi M , Saul C , Braga N M , CorreaP. H ot mate drinking and precancerouslesions of theoesophagus: an endoscopic survey in southern Brazil. Int J Cancer 1987;39:708-9.

44. Biasco G, Paganelli G M . European trials on dietary supplementation for cancer prevention. Ann N Y Acad Sci 1999;889:152-6.

45. Chang W-CL, Chapkin RS, Lupton JR. Fish oil blocks azoxymethane induced rat colon tumorigenesisby increasing cell differentiation and apoptosis rather than decreasing cell proliferation. J N utr 1998;128:491-7.

46. Le M archand L, W ilkens LR, H ankin JH, Kolonel LN , Lyu L-C. A case-control study of diet and colorectal cancer in amultiethnic population in $\mathrm{H}$ awaii (United States): lipids and foods of animal origin. C ancer $C$ auses $C$ ontrol 1997;8:637-48.

47. Estève J, Riboli E, Péquinot $G$, Terracini $B$, M erletti F, Crosignani P, AscunceN , Zubiri L, 
Blanchet F, Raymond L, Repetto F, Tuyns AJ . $D$ iet and cancers of the larynx and hypopharyns: theIARC multi-center study in southwestern Europe. $C$ ancer $C$ auses C ontrol 1996;7:240-52.

48. Fernadez E, Chatenoud L, LaVecchia C, N egri $E$, Franceschi $S$. Fish consumption and cancer risk. Am J Clin N utr 1999;70:85-90.
49. Kritchevsky D. Conjgated linoleic acid. N utr Bull 2000;25:25-7.

50. M ohan R, Sivak J, Ashton P, Russo LA, Pham $B Q$, Kasahara N, Raizman M B, Fini ME. Curcuminoids inhibit the angiogenic response stimulated by fibroblast growth factor-2, including expression of matrix metalloproteinase gelatinaseB.J Biol Chem 2000;275:10405-12. 